\title{
The "HumFlow" Project - Developing a minimal invasive measurement system for estimating energy and humidity transfer processes through building walls
}

\author{
Alessio Cavaterra ${ }^{1 *}$, Andreas Böttcher ${ }^{1}$ and Steven Lambeck ${ }^{1}$ \\ ${ }^{1}$ Department of Electrical Engineering and Information Technology, Fulda University of Applied Sciences, Germany
}

\begin{abstract}
Establishing suitable indoor climate conditions for exhibits in historical buildings is one of the main goals for curators in museums and exhibitions. Different guidelines in the field of preventive conservation provide therefore useful criteria and methods to prevent or to delay the deterioration of the objects of cultural heritage. Maintaining such a suitable indoor climate often needs the usage of expensive HVAC-equipment, whose installation occasionally damages the historical structure of such buildings and poses a problem for protection standards for historical monuments. The ongoing "HumFlow" project contributes to the solution of two problems: Firstly, avoiding damages to building structures provoked by installation of measurement devices and, secondly, gaining enough measurement data and information of humidity and energy transfer processes of walls and other surfaces by developing a minimal invasive measurement system. This paper focuses on the overall project goals, describes the test bed including experiment design, discusses the first results and provides a simple hygrothermal model of a flat wall. Therefore, it can be seen as an introduction for subsequent contributions of this project.
\end{abstract}

\section{Introduction}

Suitable indoor climate conditions in historical buildings have been discussed intensively over the last decades in the so called field of preventive conservation [1]. The latter offers guidelines and methods to prevent or to delay damages in building structures and especially to objects of cultural heritage. Most of the guidelines take the indoor temperature and the indoor humidity into account. Some proposals are discussed in [2-4] and consider maximum and minimum temperature and humidity values as well as limits for hourly or daily rates of change of these two quantities.

Some of the historical buildings are used as museums, libraries or even for exhibitions. Curators and exhibitors often struggle to comply with the temperature and humidity guidelines mentioned above. There are two reasons for this fact: historical buildings

- are usually constructed with materials like sandstone, which act as a storage for heat and moisture [5],

- are often not equipped with expensive and welltuned Heating-, Ventilation- and Air Conditioningequipment (HVAC), which could lead to the fulfilment of the guidelines.

The first reason is very important because the stored heat and moisture in the walls affect the temperature and humidity inside the building. As an example, high moisture quantities in combination with high room temperatures will not lead to a lower room humidity. Instead, the high temperature will heat up the stored moisture inside the walls, which will lead to a higher humidity inside the room [6]. These nonlinear effects are part of the hygrothermal building behaviour and need to be observed by a sensory device. Longterm observations are most desirable to gather enough information about these nonlinearities. Such a device needs to be installed on the wall without damaging the wall itself. Otherwise the requirements of valid preservation guidelines are not met. Devices based on microwave technology are able to measure the wall moisture up to $80 \mathrm{~cm}$ wall depth. These devices allow the deepest possible, non-destructive moisture measurement inside walls, but are very expensive, are affected by measurement errors and are not suitable as mobile measurement devices [7].

The second reason mentioned is the installation of HVAC-equipment. Installing HVAC-equipment occasionally harms the structure of such buildings and therefore poses a problem in terms of preservation orders. Moreover, curators and exhibitors can often not afford such expensive equipment. Retrofitting the building structure to improve the isolating characteristics is also a very expensive and time consuming task. As a result, curators and exhibitors tend to use mobile and cheap HVAC-devices in form of dehumidifiers and humidifiers, which are most likely not able to meet the indoor climate demands in a desired way.

An approach to solve this dilemma is the use of advanced control strategies in combination with affordable HVAC-equipment. To use advanced control techniques, like predictive control e.g., the hygrothermal behaviour of the building (temperature and humidity on the walls surface) needs to be observed at any time. So, the installation of measurement devices without

\footnotetext{
Corresponding author: Alessio.Cavaterra@et.hs-fulda.de
} 
damaging the building structure seems to be a solution. In other words, to establish a suitable indoor climate, given by preventive conservation guidelines, affordable measurement devices for the use of the control algorithms are needed and, at the same time, harming the building structure should be avoided. These two goals can be reached by the use of a minimal invasive measurement system. The development of such a system is the motivation of the ongoing project named "HumFlow".

This paper is organized as follows: In the subsequent section the main goals of the "HumFlow"-project are presented. Section 3 focuses on experimental design and methods. In the first part of this section the test bed including the used sensors and data acquisition concept are described in detail. After that, the modelling of the heat and moisture transfer in a flat wall is presented. This gray-box model is derived using an electrical circuit analogy. The last section concludes with some suggestions for future work to be done and some possible applications.

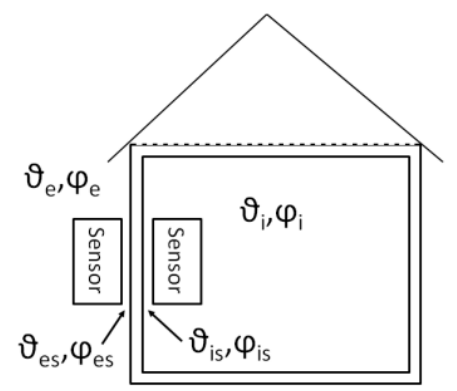

Figure 1 Humidity Measuring Approach

\section{Main Goals of "HumFlow"}

In the following the tasks of the "HumFlow" project will be briefly described. As mentioned in the introduction section, we seek to develop a minimal invasive measurement device, which is capable of sensing the surface temperature and surface humidity of walls, as well as the indoor and outdoor temperature and humidity (see figure 1). Various types of walls will be investigated concerning their hygrothermal behaviour.

Measuring the surface temperature and surface humidity of the exterior and interior wall surfaces makes the determination of the energy and moisture flow through the wall possible. Furthermore, by storing the measurement data and using system identification methods, it will be possible to identify crucial parameters like the heat transmission coefficient and the heat storage capacity of a wall. Estimating these parameters opens up new possibilities. A hygrothermal model of a room or even a whole building can be obtained by using the measurement data of multiple minimal invasive sensors. To obtain a detailed model based on physical heat and mass transfer effects, one usually ends up with partial differential equations, which are most likely nonlinear, difficult to calculate and therefore not suitable for our task. Simplifying these equations to nonlinear ordinary differential equations and gaining simpler models will give us a desirable approach.

Following this approach, the resulting model will enable the usage of model-based control methods like model predictive control [8] for mobile dehumidifiers or humidifiers. These advanced control strategies can improve the indoor climate in a suitable way, so that the mentioned guidelines are met. Because the knowledge of the hygrothermal behaviour of the building or a room is represented in these models, control engineers are able to develop energy efficient climate control strategies.

In addition to the use of these models in a control strategy, the possibility of estimating the heat and moisture distribution inside these walls by using state observers [9] is studied. Even if the estimated temperature and humidity states inside are not representing the true values, the control engineer will be able to grasp the main process gains and process time constants to derive important information regarding the potential of mould growth and other deteriorating phenomena. Predicting the indoor climate within narrow limits should no longer be a problem.

In this section the main goals and plans of the project are described. The key focus is chosen on modelling the hygrothermal behaviour of walls in a way, that it can be used for a model-based control design. The practical implementation will not be discussed in detail, because a proof of concept should be realized at first.

\section{Methods and Experimental Design}

In this section we will first describe the design of the test bed in detail. After that we will explain the methods used for modeling the hygrothermal behaviour of a flat wall.

\subsection{Test bed}

The main objective of the test bed is to gather data on the hygrothermal properties of typical wall assemblies as they are found in buildings. Therefore, the test bed has to facilitate the controlled exposure of representative wall specimens to conditions as they are commonly expected due to environmental factors. The dynamic behaviour of the temperature and relative humidity at multiple metering points as function of the respective stimulation is recorded as a time series for the applied data-driven modelling and verification methods.

\subsubsection{General structure of the test bed}

To realize the controlled exposition of the wall segments to defined stimulations, a conditioned box is used, that allows setting predefined temperature and humidity potentials, relative to the surrounding air. The wall segments (see figure 2) can be inserted through a hatch in the top, so that they form a barrier between the conditioned space and the outside air, via the open side of the box. The humidity in the conditioned space is controlled by a compact air conditioner "Schönbauer EBC10" [10], which is based on peltier technology and 
commonly used for the conditioning of display cabinets. Additionally, the air temperature can be controlled by an electric heating element. Using humidifiers and dehumidifiers, the surrounding air can be altered to the desired state optionally [11]. Refer to figure 3 for an overview.

The structure of the box is built from phenolic resin coated plywood that offers reasonable air-tightness and mechanical stability for the handling of the heavy wall elements. The box is built in the outer dimensions of $50 \times 50 \times 51 \mathrm{~cm}$ with a wall and bottom thickness of $1 \mathrm{~cm}$ and $2 \mathrm{~cm}$ to accommodate the wall elements. To maintain the portability of the test bed, wheels are mounted to the bottom of the box.

To achieve widely applicable results, three wall segments made of common construction materials (clay, sandstone, brick) were built, including layers of plaster, to replicate a representative wall structure. To define the outer dimensions, to provide an air tight seal, to avoid diffusion effects to the sides and to simplify handling, each segment is built in a welded steel frame with a detachable handle, in the dimensions $48 \times 48 \times 15 \mathrm{~cm}$.

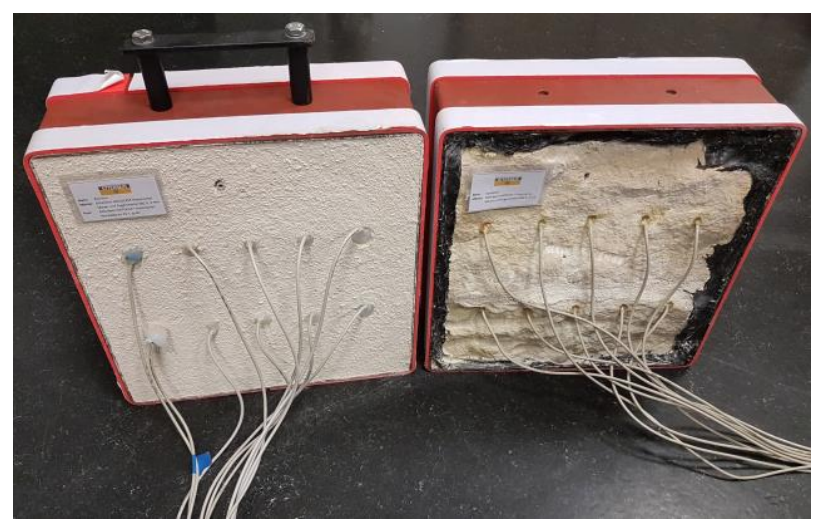

Figure 2 wall segments

\subsubsection{Sensors}

As it is the objective of the project to estimate the hygrothermal conditions in the wall based on the exterior stimulations by the temperature and humidity on both sides of the wall, those measurands need to be recorded on the wall surface and at varying depths below. Due to the varying requirements of the metering points several sensor types are used. The first sensor type is a combined humidity and surface temperature sensing unit. These custom sensing units are placed $10 \mathrm{~mm}$ in front of the wall to sense the conditions directly at the wall surface. The relative humidity at this location is recorded by an integrated capacitive type HYT321 sensor with an $\mathrm{I}^{2} \mathrm{C}$-bus-interface. In case of a temperature gradient between the sides of the wall, if the temperature between the location of the sensor and the wall surface differs, the relative humidity at this point also deviates. To compensate this systematic error, the wall surface temperature is measured using an MLX90614 infrared surface temperature sensor with $\mathrm{I}^{2} \mathrm{C}$-bus-interface. The relative humidity directly at the surface can then be determined from the reading in
$10 \mathrm{~mm}$ distance and the surface temperature, if the air in the vicinity of the surface is considered as well mixed.

Furthermore, the actual values of the humidity and temperature in varying depths of the wall are metered. For this task five combined temperature and (capacitive) relative humidity sensors are placed at equal distances through the diameter of the wall elements. For verification purposes a second row of five sensors are inserted below the first. To avoid interference, the sensors are horizontally and vertically aligned in a grid pattern with equal distances to maximize the space between each sensor and the outer frame of the wall elements. Due to their small form factor, SHT21 sensors were selected that can be interfaced via the $\mathrm{I}^{2} \mathrm{C}$-bus. A custom circuit board connects the sensors electrically to the cable and provides a certain level of thermal isolation to the $0.14 \mathrm{~mm}$ cooper conductors. The sensor assemblies are inserted in a $6 \mathrm{~mm}$ bore and sealed to the outside air. These sensors are also used to record the hygrothermal conditions in the conditioned box and the surrounding room air.

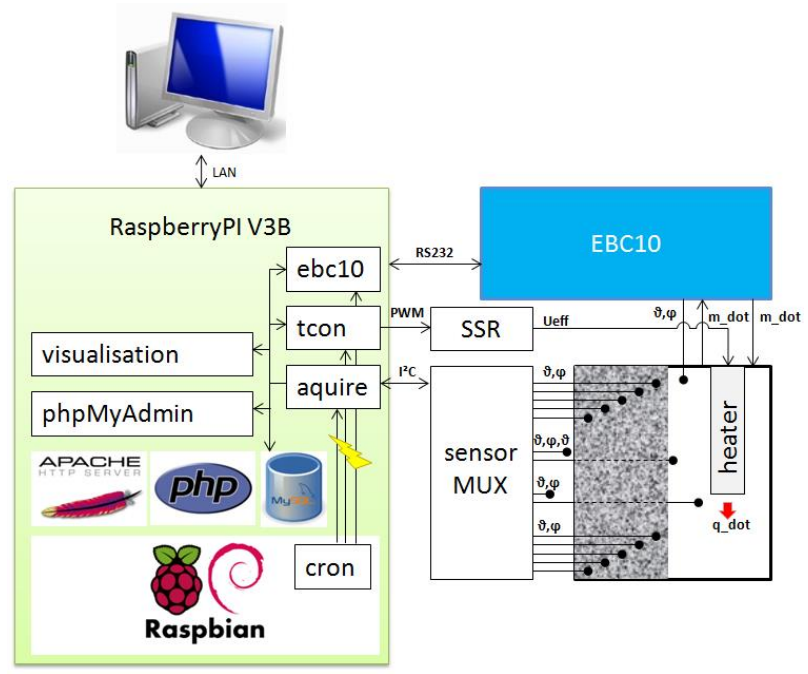

Figure 3 General Structure of the test bed

\subsubsection{Data Management and Acquisition}

Due to the relatively slow nature of the moisture absorption and temperature conduction processes, the acquired data needs to be recorded over a time-span of weeks in a defined interval. For this task, a Raspberry PI compact single-board computer is used, that provides the sensor interfacing and storage capabilities (see figure 3 ). The $\mathrm{I}^{2} \mathrm{C}$-bus-interface of the Raspberry PI is available via the expansion pin header. Since several of the used sensors share the same $\mathrm{I}^{2} \mathrm{C}$-bus-address, a circuit with two PCA9548 bus multiplexers (MUX) was designed, that spreads out the single $\mathrm{I}^{2} \mathrm{C}$-bus of the Raspberry PI to 16 individually addressable busses.

The Raspberry PI runs a version of Raspbian Linux with a MySQL database for the flexible management of the acquired data. A program, written in $\mathrm{C}$, is used for the acquisition of data in regular intervals. The scheduling service cron, integrated in the Linux OS, launches the program every minute. The program then 
selects each sensor sequentially via the $\mathrm{I}^{2} \mathrm{C}$-multiplexer, performs a measurement and reads back the acquired value. The program furthermore establishes a connection to the database and inserts the measured value with the sensor id and time the measurement was performed. The database is also used to store the nominal values for the humidity and temperature in the conditioned box. For those tasks additional programs are called by cron in regular intervals. For the temperature control, the corresponding program reads the nominal and actual temperature from the corresponding tables of the database. Then a PI-controller algorithm is executed and the duty cycle of the pulse-width-modulated signal for the solid state relay of the heating element is altered as the control value. This is performed by writing the corresponding hardware register of the PWM-peripheral unit of the Raspberry PI. The EBC10 compact air conditioner, used to control the humidity in the box, can be remotely controlled via RS232. The nominal values for the humidity are read from the database by the corresponding $\mathrm{C}$ program. This furthermore requests the nominal value from the EBC10, connected via an USBto-RS232 adapter. If the nominal values differ, the new nominal value from the database is sent to the EBC10 via RS232. For the comfortable management of the database the Raspberry PI runs the MySQL administration tool phpMyAdmin. This tool facilitates database operations using the web browser. So the nominal values for the temperature and humidity in the box and the scheduled time to activate them can be set. The recorded data can be exported in various formats like .csv and .xls.

The overall test bed is shown in figure 4 with the $\mathrm{EBC} 10$ on top of the box. The photo is taken from the open side of the box, where the surrounding air of the room simulates outside temperature and humidity conditions.

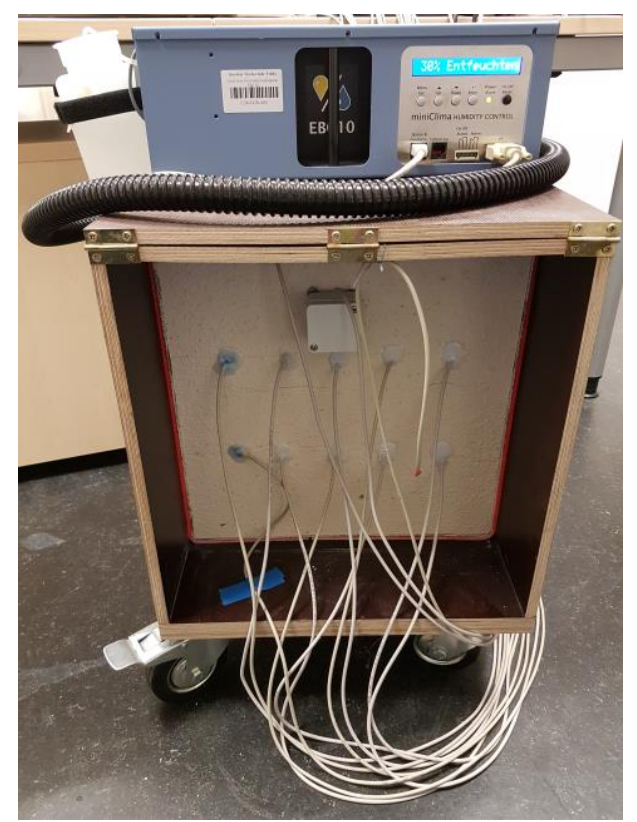

Figure 2 realized test bed
Figure 5 shows the response of a wall segment to external stimulation, recorded over the course of three weeks during the summer. The humidity in the box is controlled by the EBC10 according to a predefined trajectory, while the outside condition is altered by means of ventilation a humidification. Furthermore, the inner box temperature is increased above ambient by the integrated heating element at $\mathrm{T}=70 \mathrm{~h}$. Upon the stimulations the deepest and highest sensor (seen from outside) will be affected to a different extend, by the humidity and temperature gradient between in- and outside conditions, according to its geometrical position in the wall.

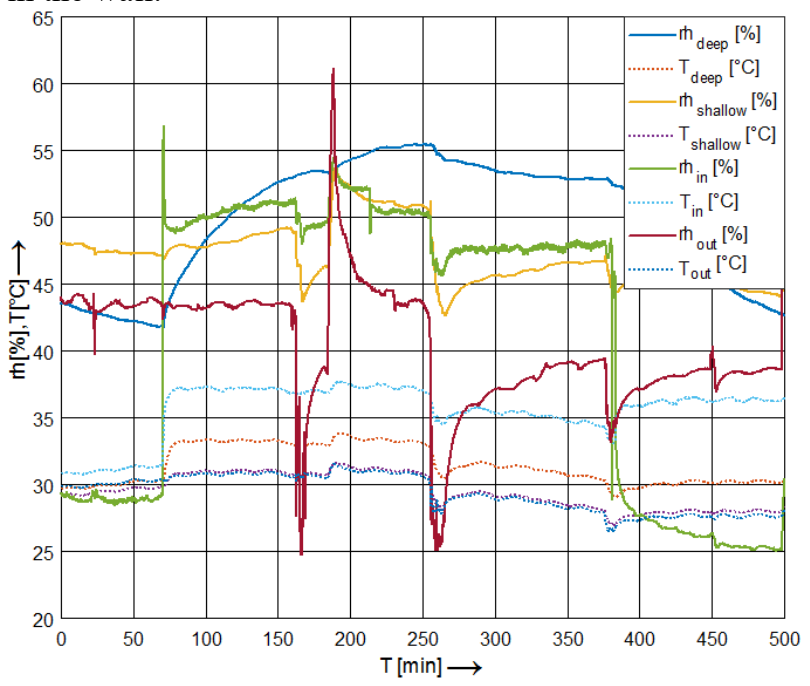

Figure 5 temperature and humidity time series

\subsection{Modelling heat and moisture transfer}

For the determination of a model of the examined wall, several techniques can be applied. First a model can be derived by setting up equations, based on the underlying physical basic principles and geometric properties. This approach requires exact knowledge of the characteristics of the used materials and the structure of the analysed wall to achieve reasonably realistic results. Depending on the level of detail during the process of deriving the model, it is usually tailored to a specific geometry and may require extensive adaptation for the utilisation in another application. Besides this so-called white box modelling approach, data-driven black box methods like artificial neural networks [12], nonlinear autoregressive exogenous models, fuzzy inference system, etc. can utilized for the derivation of the wall model. These approaches do not require the knowledge of the internal structure of the modelled system. It is later determined as a result of the estimation process. A time-series of the inputs and outputs of the system is required for this procedure, where a model is estimated that interrelates the examined state variables to the past stimulations. The process can be highly automated. A third approach is the grey box modelling scheme, which combines the approaches mentioned before. Here certain knowledge of the system's general structure is available. So a set of equations can be derived, that facilitate the simplified description of the physical processes, while still 
maintaining a relatively high level of interpretability. The model parameters can then be estimated by least square methods or something alike. Since the first principle modelling would require considerable effort and would not yield the widely applicable model and design process pursued, we will focus on black-box and grey-box models.

For the derivation of a suitable model it is important to know, that the climate inside a room depends on multiple phenomena like global radiation, outside climate, interior design and decoration, number of people inside the room, ventilation rate, thermal coupling to neighbouring rooms and many other effects which cannot be described to full extent. Just a few effects are named which are already characterized by strong nonlinear behaviour and are very difficult to measure or to describe. Even if there are possibilities to determine some of the effects, no conclusive information about the heat and moisture input, the air motion etc. will be raised. For example, estimating the number of people in the room using light barriers, will not give enough information to determine the heat input these people are emitting. Similar statements can be given for the other phenomena. In this particular application possible rearrangements in exhibition rooms with changes in the interior design and decoration of a room should be considered. This will affect the dynamical behaviour of the indoor climate in a significant way. These simple examples show, that the determination of the climate inside a room is a difficult task. However, measuring the temperature and humidity inside and outside of a room is possible using sensory devices. Additional information about the surface temperatures and surface humidity of the room enclosures - or walls to be precise - makes the modelling of the wall possible, if two sensory devices on the outside and inside of the room are aligned appropriately. Gathering this information might seem to give small profit to predict the indoor temperature and humidity conditions, but in fact this approach can be seen as one of the few possibilities which are inexpensive and provide the most beneficial outcome. Furthermore, rooms without HVAC-installations are mostly influenced by heat radiation [13]. Measuring the surface temperature and humidity of walls therefore seems to be a promising approach. In conclusion, using this approach will enable to get important information regarding the hygrothermal behaviour of a room. The previous section set the objective to derive a suitable hygrothermal model of an arbitrary wall to estimate the temperature and moisture distribution inside of it. At first, the thermal model will be described in the following subsection. The modelling of the moisture transfer will be presented in the subsequent subsection.

\subsubsection{Heat transfer in a flat wall}

It is well known, that the temperature and humidity inside a wall are functions of time with partially distributed parameters. As mentioned before, one has to deal with PDE's because the underlying heat conduction mechanism as well as the moisture transport mechanism depend on local conditions inside the wall. These PDE's are based on the heat and diffusion equation and are usually solved by a Crank-Nicholson algorithm [14]. But, as already mentioned, that approach is not in the focus of this paper. These equations can be simplified by locally discretizing them. The resulting equations are then resulting in ODE's which are easier to handle. In this case, they describe the one-dimensional heat and moisture transfer process through a wall with a predefined number of layers inside. Focusing on heat conduction mechanism, this procedure results in the so called "Beuken" model - an electrical analogy model with lumped parameters developed by Clemens Louis Beuken in 1936 [15], who exploited the fact that the heat equation and the cable equation [16] are structurally the same (see table 1).

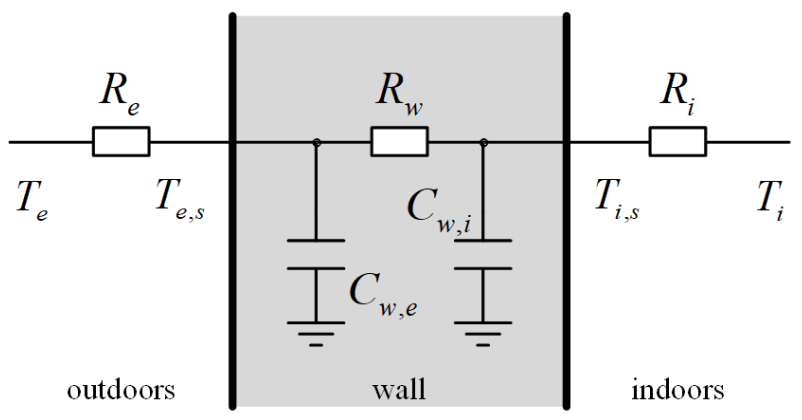

Figure 6 Electric circuit analogy for modelling the heat conduction inside a flat wall.

A graphical representation of this "Beuken" model is shown in figure 6 . The model is still used in recent research and development projects $[17,18,19]$. Figure 6 illustrates a cross-sectional area of a wall with an overlaid electrical analogy network. $T_{e}$ and $T_{i}$ represents the exterior and interior absolute temperature in Kelvin, respectively. The index " $s$ " denotes the wall surface temperatures. Thermal resistance is denoted with $R$ $(\mathrm{K} / \mathrm{W})$, while thermal heat capacity is denoted with $C$ $(\mathrm{J} / \mathrm{K}) . \quad R_{e}$ and $R_{i}$ are the reciprocal values of the convective heat transfer coefficients. $R_{w}$ is the sum of the two thermal resistances of the two wall layers. The heat capacity of each layer is denoted with $C_{w, e}$ and $C_{w, i}$. The heat flux density, which is not shown in the figure, flows from the exterior through the wall into the interior. This simple model represents a second-order ODE, which will be transformed later into a state space representation. The following two first order ODEs result after applying Kirchhoff's circuit laws:

$$
\begin{aligned}
& C_{w, e} d T_{e . s} / d t=-\left(1 / R_{e}+1 / R_{w}\right) T_{e, s}+T_{i, s} / R_{w}+T_{e} / R_{e} \\
& C_{w, i} d T_{i . s} / d t=-\left(1 / R_{i}+1 / R_{w}\right) T_{i, s}+T_{e, s} / R_{w}+T_{i} / R_{i}
\end{aligned}
$$

As already stated, the wall surface temperatures as well as the outdoor and the indoor temperatures are measured by the sensory device. The latter are interpreted as the input variables in this context. The surface temperatures are modeled as the systems state and output variables.

The thermal resistances and the thermal heat capacities are assumed to be constant within their 
assigned wall layer. Furthermore, the effect of moisture transport on the heat transfer mechanism is neglected.

\subsubsection{Extending the model with moisture transfer}

Most of the building materials are porous. In the pores water (or moisture in this context) can exist in the gaseous, liquid or solid state. Effects caused by ice inside the walls are neglected. The main mechanisms of moisture transfer are water vapour diffusion and capillary suction, which depends on the moisture content of the material. Moisture storage inside porous materials is often described by the moisture retention function (Fig. 7), which is also known as "sorption isotherm".

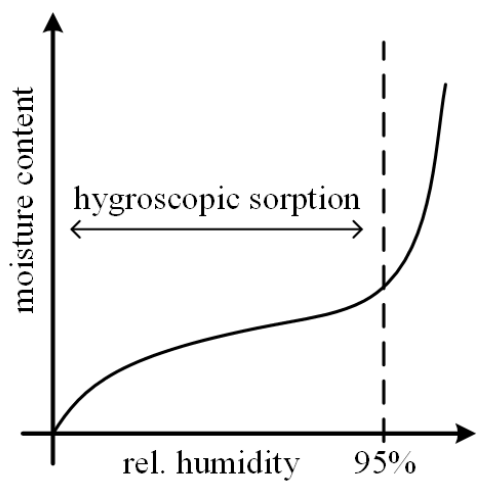

Figure 7 Moisture retention function or sorption isotherm for a porous material

Between the $0 \% \mathrm{rH}$ to $95 \% \mathrm{rH}$ relative humidity range the so called hygroscopic sorption range - the moisture transport is mainly caused by vapour diffusion. For higher values of relative humidity $(>95 \%)$ the amount of moisture content in the regarded material per relative humidity value will increase faster than before. This region is called the capillary sorption region, because the main moisture transport mechanism relies on capillary suction [20]. The capillary effects will be neglected as well as adsorption-desorption hysteresis effects, which occur in moisture retention functions. For further information regarding the capillary effects, the hysteresis effects and the moisture retention function and its meaning for modelling heat and moisture processes, refer to $[20,21,22]$.

Table 1 Analogy between heat and diffusion

\begin{tabular}{|c|c|}
\hline Heat & Diffusion \\
\hline Fourier's law & Fick's first law of diffusion \\
$q=-k \frac{\partial T}{\partial x}$ & $j=-D \frac{\partial \varphi}{\partial x}$ \\
\hline Heat equation & $\begin{array}{c}\text { Diffusion equation or } \\
\text { Fick's second law } \\
\frac{\partial T}{\partial t}=\alpha \frac{\partial^{2} T}{\partial x^{2}}\end{array}$ \\
$\frac{\partial \varphi}{\partial t}=D^{\prime} \frac{\partial^{2} \varphi}{\partial x^{2}}$ \\
\hline \multicolumn{2}{|c|}{ Cable equation [13] $\frac{\partial V}{\partial t}=\frac{1}{c_{l}} \frac{\partial^{2} V}{\partial x^{2}}-\frac{V}{r_{m}}$} \\
\hline
\end{tabular}

Comparing the partial differential equations of moisture transfer processes with the ones describing heat transfer processes in table 1, we can see, that there is some kind of analogy: Fick's law is similar to Fourier' law and the diffusion equation is structurally the same as the heat equation. As already stated in the previous subsection, Beuken exploited the analogy between heat equation and cable equation - therefore, the moisture transfer process through a flat wall can be approximated by using an electrical circuit analogy model as well. This lumped parameter model approach is not new. In $[17,20]$ a lumped-parameter model is mentioned, too.

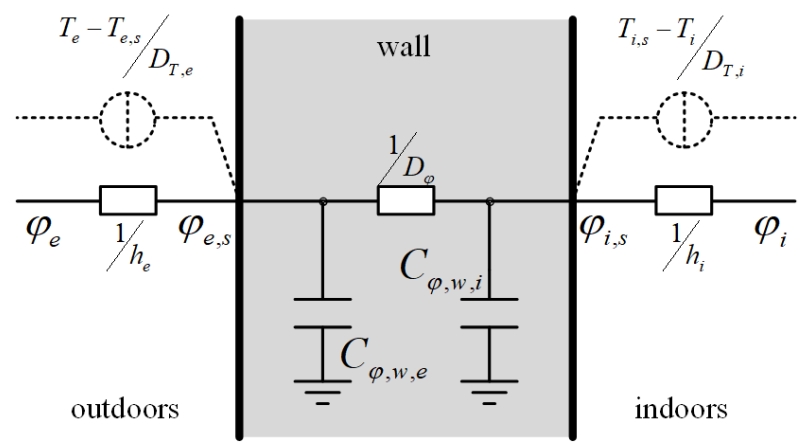

Figure 8 Electric circuit analogy for moisture transfer

While the temperature is the driving potential for the heat flux density in the heat transfer case, the driving potential in the moisture transfer case is physically the water vapour pressure. In the literature, there are mainly three different driving potentials chosen. Some authors use moisture content [22], some use vapour pressure [20, $21]$, some others use relative humidity $[19,23]$. In the present case it is favourable to use the relative humidity as a driving potential because (i) the relative humidity on the wall surfaces on the inside and the outside of the building is measured anyway and (ii) the relative humidity is continuous between the material boundaries while the other quantities are usually discontinuous [20].

In contrast to the heat transfer model presented in the previous subsection the effects of temperature on the moisture transport process will not be ignored. Temperature has a strong influence on the water vapour pressure, which in turn has a great effect on the relative humidity. This dependency is included by adding a current source and a current sink into the electrical analogy model, see figure 8 . The source and the sink represent the moisture flux caused by temperature gradients on the exterior and interior of the wall and contain the associated thermal diffusion coefficients, denoted by $D_{T, e}$ and $D_{T, i}(\mathrm{~K} \cdot \mathrm{s} / \mathrm{kg})$. The other diffusion coefficients are directly linked to relative humidity and are also stated with $D$ and the associated indexes. The convective mass transfer coefficients are denoted by $h_{e}$ and $h_{i}(\mathrm{~kg} / \mathrm{s} / \% \mathrm{rh})$. These are linked to the convective heat transfer coefficients by the Lewis relation [24]. The ability to store moisture of the material is denoted with $C$ $(\mathrm{kg} / \% \mathrm{rh})$. These sorption capacities $\left(\mathrm{kg} / \mathrm{m}^{3}\right)$ can be approximately specified by constructing a point-wise tangent of the sorption isotherm of the porous material [20] and multiplying the slope with the material volume. 
Two ordinary differential equations can now be derived by Kirchhoffs' circuit laws, similar to the procedure in the previous section for the thermal model. In the following, the overall one-dimensional heat and moisture state-space model of a flat wall will be presented. State Space models are a matrix notation of first order ODEs, which can be described by:

$$
\begin{aligned}
d \boldsymbol{x}(t) / d t & =\boldsymbol{A} \boldsymbol{x}(t)+\boldsymbol{B} \boldsymbol{u}(t) \\
\boldsymbol{y}(t) & =\boldsymbol{C} \boldsymbol{x}(t)+\boldsymbol{D} \boldsymbol{u}(t)
\end{aligned}
$$

The vector $\mathbf{x}(\mathrm{t})$ contains the state variables, while the systems inputs are stored in $\mathbf{u}(\mathrm{t})$. The outputs are denoted by $\mathbf{y}(\mathrm{t})$. In the case, which is regarded in this paper, the state vector contains all measured surface quantities

$$
\boldsymbol{x}(t)=\left[\begin{array}{llll}
T_{e, s} & T_{i, s} & \varphi_{e, s} & \varphi_{i, s}
\end{array}\right]^{T},
$$

the input vector consists of the interior and exterior temperature and humidity

$$
\boldsymbol{u}(t)=\left[\begin{array}{llll}
T_{e} & T_{i} & \varphi_{e} & \varphi_{i}
\end{array}\right]^{T},
$$

and the output is simply the state, $\mathbf{y}(\mathrm{t})=\mathbf{x}(\mathrm{t})$. Note, that every element of the matrix $\mathbf{D}$ is zero and $\mathbf{C}$ is the $4 \times 4$ identity matrix. The system matrix $\mathbf{A}$ and the input matrix $\mathbf{B}$ are given by

$$
\begin{aligned}
\boldsymbol{A} & =\left[\begin{array}{cccc}
a & \frac{1}{C_{w, e} R_{w}} & 0 & 0 \\
\frac{1}{C_{w, i} R_{w}} & b & 0 & 0 \\
\frac{-D_{T, e}}{C_{\varphi, w, e}} & 0 & c & \frac{D_{\varphi}}{C_{\varphi, w, e}} \\
0 & \frac{D_{T, i}}{C_{\varphi, w, i}} & \frac{D_{\varphi}}{C_{\varphi, w, i}} & d
\end{array}\right], \\
\boldsymbol{B} & =\left[\begin{array}{cccc}
\frac{1}{C_{w, e} R_{e}} & 0 & 0 & 0 \\
0 & \frac{1}{C_{w, i} R_{i}} & 0 & 0 \\
\frac{D_{T, e}}{C_{\varphi, w, e}} & 0 & \frac{h_{e}}{C_{\varphi, w, e}} & 0 \\
0 & \frac{-D_{T, i}}{C_{\varphi, w, i}} & 0 & \frac{h_{i}}{C_{\varphi, w, i}}
\end{array}\right] .
\end{aligned}
$$

Where

$$
\begin{aligned}
& a=-\left(1 / R_{e}+1 / R_{w}\right) / C_{w, e} \\
& b=-\left(1 / R_{i}+1 / R_{w}\right) / C_{w, i} \\
& c=-\left(h_{e}+D_{\varphi}\right) / C_{\varphi, w, e} \\
& d=-\left(h_{i}+D_{\varphi}\right) / C_{\varphi, w, i} .
\end{aligned}
$$

The matrices $\mathbf{A}, \mathbf{B}, \mathbf{C}$ and $\mathbf{D}$ are usually assumed to be constant. This assumption might be restrictive, because the given coefficients depend on temperature and humidity value. See for example the slope of the sorption isotherm in figure 7 , which acts on the value of the sorption capacities $C_{\varphi, w, e}$ and $C_{\varphi, w, i}$. Note, that the heat and moisture model described by eq. (3) - (12) is fully observable and controllable in the sense of Kalman [9]. Therefore, these unknown coefficients can be identified by using a least squares method, LevenbergMarquardt algorithm or a similar method. Knowing these parameters, the prediction of the future wall surface temperatures and humidities is possible, if future values of $T_{e}, T_{i}, \varphi_{e}$ and $\varphi_{i}$ are known. This task can be further simplified, assuming constant $T_{i}$ and $\varphi_{i}$.

\section{Conclusion}

In this paper we present the ongoing research and development of the project "HumFlow". The main goal of the "HumFlow" project is the development of a minimal invasive sensory device, which is capable of measuring and predicting the relative humidity and temperature on the surface of the exterior and interior of a wall. Based on this information, we are able to control the climate conditions inside historical buildings in order to prevent possible deterioration of cultural heritage and to meet the climate guidelines given by preventive conservation.

In order to test our ideas, we build a test bed for experiments, consisting of different building materials which are interchangeable. A Raspberry Pi single board computer is used for data acquisition and storage.

To predict the heat and moisture transfer processes through arbitrary flat walls we develop a lumped parameter state space model using the electrical circuit analogy suggested by Beuken. This fourth order model is fully controllable and observeable in the sense of Kalman, so that a parameter estimation will be possible. A drawback of the approach is the simple model structure, which might not reflect the true heat and moisture transfer inside the wall.

Nevertheless, this approach opens up new possibilities. As mentioned before, long term monitoring is beneficial to understand the hygrothermal behaviour of a building. This will be helpful to further improve the indoor climate conditions of buildings. In combination with predictive algorithms, this device enables the possibility to plan heating and humidifying strategies and saving up energy resources. This approach can also be useful for existing HVAC equipment in modern buildings. Installing such a device before refurbishing a building enclosure, might help in making the right decisions. Many other aspects can be thought of.

Because it is an ongoing project, "HumFlow" still offers a lot of work to be done in the future. The prototype needs further development regarding the surface mounting kit and the radio transmission of the measurement data. We need to gather data on real buildings and we need to establish a wireless communication network so that the devices are able to send their data to a local server. The nonlinear coefficients need to be modeled with advanced methods. One way to solve the latter is the usage of TakagiSugeno fuzzy models [25]. If this extension of the above hygrothermal model is done, a thorough validation of the model is planned. Results will be presented in a subsequent paper.

We would like to thank the Hessian State Ministry for Higher Education, Research and the Arts for funding the "HumFlow"-project within the "Forschung für die Praxis"campaign (engl. "research for the practice"). 


\section{References}

1. Jeberien, A. and Knaut, M., Preventive Conservation (University of Applied Sciences Berlin, 2007)

2. European Committee for Standardisation, EN 15757 Conservation of Cultural Property - Specifications for temperature and relative humidity to limit climate-induced mechanical damage in organic hygroscopic materials, Brussels (2010)

3. L. Bratasz, Climate for Collections - Standards and Uncertainties, Allowable microclimatic variations in museums and historic buildings: reviewing the guidelines, p. 11-20, (2013)

4. R. Kilian, T. Vyhlidal and T. Broström, Developments in climate control of historic buildings, Fraunhofer IRB (2010)

5. A. Turkington and T. Paradise, Geomorphology, Sandstone weathering: a century of research and innovation, 67, Issues 1-2, p. 229-253 (2005)

6. T. Padfield, The role of absorbent building materials in moderating changes of relative humidity, Department of Structural Engineering and Materials, Lyngby, Technical University of Denmark, 150 (1998)

7. R. Wernecke, Industrielle Feuchtemessung: Grundlagen, Messmethoden, technische Anwendungen. (Wiley, 2003)

8. E.F. Camacho and C. Bordons, Model Predictive Control, 2 (2003)

9. R. Isermann, Digital Control Systems, Fundamentals, deterministic control, 1 (1989)

10. A. Köhler et al. Proceedings of the 11th AALEConference, Entwicklung eines Versuchsstands zur dynamischen Sollwertanpassung und Regelung der relativen Feuchte in einer Vitrine, p. 305-314 (2014)

11. A. Cavaterra et al. Proceedings of the 12th AALEConference, Multifunktionale Leitkomponente zur prototypischen Entwicklung von Regelungssystemen, p. 327-331 (2015)

12. T. Lu and M. Viljanen, Neural Computing and Applications, Prediction of indoor temperature and relative humidity using neural network models: model comparison. 18, 4 (2009)

13. S. Völkner and E. Reyer, Zum Einfluss räumlich begrenzter Diskontinuitäten auf die zeitabhängige Feuchteverteilung in Außenwänden, Fraunhofer IRB (2003)

14. European Committee for Standardisation, EN 15026 Hygrothermal performance of building components and building elements - Assessment of moisture transfer by numerical simulation, Brussels (2007)

15. C.L. Beuken, Wärmeverluste bei periodisch betriebenen Öfen, $\mathrm{PhD}$ thesis, Freiburg University (1936)

16. E.J. López-Sánchez and J.M. Romero, Cable equation for general geometry, Physical Review E 95.2 (2017)
17. R. Kramer, J.v. Schijndel and H. Schellen, Climate for Collections - Standards and Uncertainties, Inverse modelling of climate responses of monumental buildings, p.425-437 (2013)

18. D. Sierociuk et al., Applied Mathematics and Computation, Diffusion process modelling by using fractional-order models, 257, p. 2-11 (2015)

19. C. Arnold, Entwicklung fuzzybasierter Leitkomponenten für das Klimamanagement in der präventiven Konservierung, $\mathrm{PhD}$ thesis, Springer Vieweg (2013)

20. F. Tariku, K. Kumaran and P- Fazio, Int. J. Heat Mass Transf., Transient model for coupled heat, air and moisture transfer through multi-layered porous media, 53, p. 3035-3044 (2010)

21. Y. Chen and S. Wang, Building and Environment, Transfer function model and frequency domain validation of moisture sorption in air-conditioned buildings, 36, p. 579-588 (2001)

22. X. Lü, Energy and Buildings, Modelling of heat and moisture transfer in buildings I. Model program, 34, p. 1033-1043 (2002)

23. N. Mendes and P.C. Philippi, Int. J. Heat Mass Transf., A method for predicting heat and moisture transfer through multilayered walls based on temperature and moisture content gradients, $\mathbf{4 8 , \mathrm { p } .}$ 37-51 (2005)

24. Y. Liu et al., Energy and Buildings, Effect of moisture transfer on internal surface temperature, 60, p. 83-91 (2013)

25. T. Takagi and M. Sugeno, IEEE Transactions on Systems, Man and Cybernetics, Fuzzy identification of systems and its applications to modelling and control, SMC-15, No. 1, p. 116-132 (1985) 